\title{
A CONTINUATION RESULT FOR FORCED OSCILLATIONS OF CONSTRAINED MOTION PROBLEMS WITH INFINITE DELAY
}

\author{
PIERLUIGI BENEVIERI, ALESSANDRO CALAMAI, MASSIMO FURI, AND MARIA PATRIZIA PERA
}

\begin{abstract}
We prove a global continuation result for $T$-periodic solutions of a $T$-periodic parametrized second order retarded functional differential equation on a boundaryless compact manifold with nonzero Euler-Poincaré characteristic. The approach is based on the fixed point index theory for locally compact maps on ANRs. As an application, we prove the existence of forced oscillations of retarded functional motion equations defined on topologically nontrivial compact constraints. This existence result is obtained under the assumption that the frictional coefficient is nonzero, and we conjecture that it is still true in the frictionless case.
\end{abstract}

\section{INTRODUCTION}

Let $X \subseteq \mathbb{R}^{s}$ be a smooth boundaryless manifold, and let $F: \mathbb{R} \times C((-\infty, 0], X) \rightarrow \mathbb{R}^{s}$ be a continuous map such that

$$
F(t, \varphi) \in T_{\varphi(0)} X, \quad \forall(t, \varphi) \in \mathbb{R} \times C((-\infty, 0], X),
$$

where, given $q \in X, T_{q} X \subseteq \mathbb{R}^{s}$ denotes the tangent space of $X$ at $q$. Any tangent vector field with this property will be called a functional field on $X$.

Assume that $F$ is $T$-periodic in the first variable and consider the following retarded functional motion equation on $X$, depending on a parameter $\lambda \geq 0$ :

$$
x_{\pi}^{\prime \prime}(t)=\lambda\left(F\left(t, x_{t}\right)-\varepsilon x^{\prime}(t)\right),
$$

where $x_{\pi}^{\prime \prime}(t)$ stands for the tangential part of the acceleration $x^{\prime \prime}(t) \in \mathbb{R}^{s}$ at the point $x(t) \in X, x_{t}$ is the function $\theta \mapsto x(t+\theta)$, and $\varepsilon \geq 0$ is the frictional coefficient. Given $\lambda \geq 0$, any $T$-periodic solution of (1.1) is called a forced oscillation corresponding to the value $\lambda$ of the parameter. Notice that when $\lambda=0$ the above equation reduces to the so-called inertial equation and one obtains the geodesics of $X$ as solutions.

A pair $(\lambda, x)$, with $\lambda \geq 0$ and $x: \mathbb{R} \rightarrow X$ a forced oscillation of (1.1) corresponding to $\lambda$, is called a $T$-forced pair of (1.1). The set of $T$-forced pairs is regarded as a subset of $[0,+\infty) \times C_{T}^{1}(X)$, where $C_{T}^{1}(X)$ is the metric subspace of the Banach space $C_{T}^{1}\left(\mathbb{R}^{s}\right)$ of the $T$-periodic $C^{1}$ maps from $\mathbb{R}$ to $X$.

Given $q \in X$, we denote by $\bar{q} \in C_{T}^{1}(X)$ the constant map $t \mapsto q$, and we call trivial $T$-forced pair a pair of the form $(0, \bar{q})$. An element $q_{0} \in X$ will be called a bifurcation point of the equation (1.1) if every neighborhood of $\left(0, \bar{q}_{0}\right)$ in $[0,+\infty) \times C_{T}^{1}(X)$ contains a nontrivial $T$-forced pair. We point out that any nontrivial $T$-forced pair $(\lambda, x)$ of (1.1), sufficiently close to $\left(0, \bar{q}_{0}\right)$, must have $\lambda>0$. This is due to the fact that there are no nontrivial closed geodesics too close to a given point in a Riemannian manifold.

In this paper we investigate the structure of the set of $T$-forced pairs of (1.1). Our main result, Theorem 4.1 below, states that if $X$ is compact with nonzero Euler-Poincaré characteristic, and if $F$ has bounded image and verifies a suitable Lipschitz-type assumption, then there exists an unbounded

2000 Mathematics Subject Classification. 34K13, 37C25, 34C40, 70K42.

Key words and phrases. Retarded functional differential equations, periodic solutions, fixed point index theory, motion problems on manifolds. 
connected branch of nontrivial $T$-forced pairs whose closure intersects the set of the trivial $T$-forced pairs (necessarily at some bifurcation point).

We stress that, when $\varepsilon>0$, the bifurcating branch given in Theorem 4.1 is unbounded with respect to the parameter $\lambda$. On the other hand, if $\varepsilon=0$ we believe that the same result is true but we are not able to prove it. As a consequence of this observation we get (see Corollary 4.2 below) that the retarded functional motion equation

$$
x_{\pi}^{\prime \prime}(t)=F\left(t, x_{t}\right)-\varepsilon x^{\prime}(t)
$$

admits at least one forced oscillation, provided that the frictional coefficient $\varepsilon$ is positive. This existence theorem (which has been already proved in [5]) generalizes results given in 2] and [4 for equations with constant time lag (see also [11] for the undelayed case). On the opposite, in the frictionless case, the existence of an unbounded bifurcating branch is not sufficient to guarantee the existence of a forced oscillation of the equation

$$
x_{\pi}^{\prime \prime}(t)=F\left(t, x_{t}\right) .
$$

As far as we know, the problem of the existence of forced oscillations of (1.2) is still open, even in the undelayed case. An affirmative answer, in the undelayed situation, regarding the special constraint $X=S^{2}$ (the spherical pendulum) can be found in [12] (see also [14] for the extension to the case $X=S^{2 n}$ ). Let us point out that Theorem 4.1 below will be crucial for further investigation of the question whether or not the forced spherical pendulum admits forced oscillations in the retarded functional case.

To get our main result, we consider a first order retarded functional differential equation (RFDE) on the tangent bundle $T X \subseteq \mathbb{R}^{2 s}$, which is equivalent to the second order equation (1.1). More precisely, in the first and preliminary part of the paper we study first order parametrized RFDEs of the type

$$
x^{\prime}(t)=f\left(\lambda, t, x_{t}\right),
$$

where $f:[0,+\infty) \times \mathbb{R} \times C((-\infty, 0], M) \rightarrow \mathbb{R}^{k}$ is a parametrized functional field, $T$-periodic in the second variable, on a smooth manifold $M \subseteq \mathbb{R}^{k}$, possibly with boundary. In Corollary 3.6 below we prove a global continuation result for equation (1.3) under the assumption that $M$ is compact with nonzero Euler-Poincaré characteristic, $f$ is inward along the boundary (when $\partial M \neq \emptyset$ ), has bounded image and verifies a suitable Lipschitz-type assumption. Such a result is obtained by applying the classical fixed point index theory for locally compact maps on ANRs to a sort of Poincaré $T$-translation operator acting in the Banach space $C\left([-T, 0], \mathbb{R}^{k}\right)$. We point out that the continuation result for the first order equation (1.3) involves manifolds with boundary. This fact will be crucial in the application of such a result to second order equations, as it will be clear in the proof of Theorem 4.1

Among the wide bibliography on RFDEs in Euclidean spaces we refer to the works of Gaines and Mawhin [15], Nussbaum [25, 26] and Mallet-Paret, Nussbaum and Paraskevopoulos [21]. For RFDEs on manifolds we cite the papers of Oliva [27, 28. For general reference we suggest the monograph by Hale and Verduyn Lunel [18.

\section{Preliminaries}

2.1. RFDE. Let $M$ be an arbitrary subset of $\mathbb{R}^{k}$. We recall the notions of tangent cone and tangent space of $M$ at a given point $p$ in the closure $\bar{M}$ of $M$. The definition of tangent cone is equivalent to the classical one introduced by Bouligand in 7 . 
Definition 2.1. A vector $v \in \mathbb{R}^{k}$ is said to be inward to $M$ at $p \in \bar{M}$ if there exist two sequences $\left\{\alpha_{n}\right\}$ in $[0,+\infty)$ and $\left\{p_{n}\right\}$ in $M$ such that

$$
p_{n} \rightarrow p \quad \text { and } \quad \alpha_{n}\left(p_{n}-p\right) \rightarrow v .
$$

The set $C_{p} M$ of the inward vectors to $M$ at $p$ is called the tangent cone of $M$ at $p$. The tangent space $T_{p} M$ of $M$ at $p$ is the vector subspace of $\mathbb{R}^{k}$ spanned by $C_{p} M$. A vector $v$ of $\mathbb{R}^{k}$ is said to be tangent to $M$ at $p$ if $v \in T_{p} M$.

To simplify some statements and definitions we put $C_{p} M=T_{p} M=\emptyset$ whenever $p \in \mathbb{R}^{k}$ does not belong to $\bar{M}$ (this can be regarded as a consequence of Definition 2.1 if one replaces the assumption $p \in \bar{M}$ with $p \in \mathbb{R}^{k}$ ). Observe that $T_{p} M$ is the trivial subspace $\{0\}$ of $\mathbb{R}^{k}$ if and only if $p$ is an isolated point of $M$. In fact, if $p$ is a limit point, then, given any $\left\{p_{n}\right\}$ in $M \backslash\{p\}$ such that $p_{n} \rightarrow p$, the sequence $\left\{\alpha_{n}\left(p_{n}-p\right)\right\}$, with $\alpha_{n}=1 /\left\|p_{n}-p\right\|$, admits a convergent subsequence whose limit is a unit vector.

One can show that in the special and important case when $M$ is a smooth manifold with (possibly empty) boundary $\partial M$ (a $\partial$-manifold for short), this definition of tangent space is equivalent to the classical one (see for instance [22, [17]). Moreover, if $p \in \partial M, C_{p} M$ is a closed half-space in $T_{p} M$ (delimited by $T_{p} \partial M$ ), while $C_{p} M=T_{p} M$ if $p \in M \backslash \partial M$.

Let, as above, $M$ be a subset of $\mathbb{R}^{k}$. We denote by $D$ a nontrivial closed real interval with $\max D=0$; that is, $D$ is either $(-\infty, 0]$ or $[-r, 0]$ with $r>0$. By $C(D, M)$ we mean the metrizable space of the $M$-valued continuous functions defined on $D$ with the topology of the uniform convergence on compact subintervals of $D$.

Given a continuous function $x: J \rightarrow M$, defined on a real interval $J$, and given $t \in \mathbb{R}$ such that $t+D \subseteq J$, we adopt the standard notation $x_{t}: D \rightarrow M$ for the function defined by $x_{t}(\theta)=x(t+\theta)$.

Let $h: \mathbb{R} \times C(D, M) \rightarrow \mathbb{R}^{k}$ be a continuous map. We say that $h$ is a functional field on $M$ if $h(t, \varphi) \in$ $T_{\varphi(0)} M$ for all $(t, \varphi) \in \mathbb{R} \times C(D, M)$. In particular, $h$ will be said inward (to $\left.M\right)$ if $h(t, \varphi) \in C_{\varphi(0)} M$ for all $(t, \varphi)$. If $M$ is a closed subset of a boundaryless smooth manifold $N \subseteq \mathbb{R}^{k}$, we will say that $h$ is away from $N \backslash M$ if $h(t, \varphi) \notin C_{\varphi(0)}(N \backslash M)$ for all $(t, \varphi) \in \mathbb{R} \times C(D, M)$. Notice that this condition is satisfied whenever the point $\varphi(0) \in M$ is not in the topological boundary of $M$ relative to $N$ since, in that case, $C_{\varphi(0)}(N \backslash M)=\emptyset$.

Let us consider a retarded functional differential equation ( $R F D E$ for short) of the type

$$
x^{\prime}(t)=h\left(t, x_{t}\right),
$$

where $h: \mathbb{R} \times C(D, M) \rightarrow \mathbb{R}^{k}$ is a functional field on $M$.

By a solution of (2.1) we mean a continuous function $x: J \rightarrow M$, defined on a real interval $J$ with $\inf J=-\infty$, which verifies eventually the equality $x^{\prime}(t)=h\left(t, x_{t}\right)$. That is, $x$ is a solution of (2.1) if there exists $\bar{t}$, with $-\infty \leq \bar{t}<\sup J$, such that $x$ is $C^{1}$ on the subinterval $(\bar{t}, \sup J)$ of $J$ and verifies $x^{\prime}(t)=h\left(t, x_{t}\right)$ for all $t \in(\bar{t}, \sup J)$.

Observe that, when $D=[-r, 0]$, there is a one-to-one correspondence between our notion of solution and the classical one which can be found e.g. in [18] (see also [27]). The correspondence is the one that assigns to any solution of (2.1) its restriction to the interval $[\bar{t}-r, \sup J)$.

Remark 2.2. We stress that it is possible to associate to any equation of the form (2.1) with $D=[-r, 0]$ an equivalent equation of the same type with $D=(-\infty, 0]$. In other words, given a functional field $h: \mathbb{R} \times C([-r, 0], M) \rightarrow \mathbb{R}^{k}$, there exists a functional field $g: \mathbb{R} \times C((-\infty, 0], M) \rightarrow \mathbb{R}^{k}$ such that the equation

$$
x^{\prime}(t)=g\left(t, x_{t}\right)
$$


has the same set of solutions as (2.1). To see this, it is enough to define $g: \mathbb{R} \times C((-\infty, 0], M) \rightarrow \mathbb{R}^{k}$ by

$$
g(t, \varphi)=h\left(t,\left.\varphi\right|_{[-r, 0]}\right)
$$

for any $(t, \varphi) \in \mathbb{R} \times C((-\infty, 0], M)$.

As a consequence of Remark 2.2 it is not restrictive to study the broader class of RFDE's of the type

$$
x^{\prime}(t)=g\left(t, x_{t}\right),
$$

where $g: \mathbb{R} \times C((-\infty, 0], M) \rightarrow \mathbb{R}^{k}$ is a functional field on $M$. Therefore, from now on we will focus on this kind of equations.

2.2. Initial value problem. We are now interested in the following initial value problem:

$$
\begin{cases}x^{\prime}(t)=g\left(t, x_{t}\right), & t>0 \\ x(t)=\eta(t), & t \leq 0\end{cases}
$$

where $M$ is a subset of $\mathbb{R}^{k}, g: \mathbb{R} \times C((-\infty, 0], M) \rightarrow \mathbb{R}^{k}$ is a functional field on $M$, and $\eta:(-\infty, 0] \rightarrow M$ is a continuous map.

A solution of problem (2.3) is a solution $x: J \rightarrow M$ of (2.2) such that $\sup J>0, x^{\prime}(t)=g\left(t, x_{t}\right)$ for $t>0$, and $x(t)=\eta(t)$ for $t \leq 0$.

The following technical lemma regards the existence of a persistent solution of problem (2.3).

Lemma 2.3 ([5]). Let $M$ be a compact subset of a boundaryless smooth manifold $N \subseteq \mathbb{R}^{k}$, and $g$ a functional field on $M$ which is away from $N \backslash M$. Suppose that $g$ is bounded. Then problem (2.3) admits at least one solution defined on the whole real line.

From now on $M$ will be a compact $\partial$-manifold in $\mathbb{R}^{k}$. In this case one may regard $M$ as a subset of a smooth boundaryless manifold $N$ of the same dimension as $M$ (see e.g. 19, 23]). It is not hard to show that a functional field $g$ on $M$ is away from the complement $N \backslash M$ if and only if it is strictly inward; meaning that $g$ is inward and $g(t, \varphi) \notin T_{\varphi(0)} \partial M$ for all $(t, \varphi) \in \mathbb{R} \times C((-\infty, 0], M)$ such that $\varphi(0) \in \partial M$.

We say that a subset $Q$ of $C((-\infty, 0], M)$ is a brush if there exists $\sigma \leq 0$ such that

$$
\varphi(\theta)=\psi(\theta), \quad \theta \leq \sigma
$$

for all $\varphi, \psi \in Q$. We will make the following assumption:

(H) Given $\bar{t}>0$ and any compact brush $Q$ of $C((-\infty, 0], M)$, there exists $L \geq 0$ such that

$$
\|g(t, \varphi)-g(t, \psi)\| \leq L \sup _{s \leq 0}\|\varphi(s)-\psi(s)\|
$$

for all $t \in[0, \bar{t}]$ and $\varphi, \psi \in Q$.

The following proposition regards existence and uniqueness of solutions of problem (2.3) in the case when $g$ is inward, bounded, and verifies $(\mathrm{H})$.

Proposition 2.4 ([5]). Let $M \subseteq \mathbb{R}^{k}$ be a compact $\partial$-manifold and $g$ an inward functional field on $M$. Suppose that $g$ is bounded. Then, problem (2.3) admits a solution defined on the whole real line. Moreover, if $g$ verifies $(H)$, then the solution is unique. 
2.3. Fixed point index. Here we summarize the main properties of the fixed point index in the context of absolute neighborhood retracts (ANRs). Let $X$ be a metric ANR and consider a locally compact (continuous) $X$-valued map $k$ defined on a subset $\mathcal{D}(k)$ of $X$. Given an open subset $U$ of $X$ contained in $\mathcal{D}(k)$, if the set of fixed points of $k$ in $U$ is compact, the pair $(k, U)$ is called admissible. It is known that to any admissible pair $(k, U)$ we can associate an integer $\operatorname{ind}_{X}(k, U)$ - the fixed point index of $k$ in $U$ - which satisfies properties analogous to those of the classical Leray-Schauder degree [20]. The reader can see for instance [6], [16], 24] or [26] for a comprehensive presentation of the index theory for ANRs. As regards the connection with the homology theory we refer to standard algebraic topology textbooks (e.g. [8], [29]).

We summarize for the reader's convenience the main properties of the index.

i) (Existence) If $\operatorname{ind}_{X}(k, U) \neq 0$, then $k$ admits at least one fixed point in $U$.

ii) (Normalization) If $X$ is compact, then $\operatorname{ind}_{X}(k, X)=\Lambda(k)$, where $\Lambda(k)$ denotes the Lefschetz number of $k$.

iii) (Additivity) Given two disjoint open subsets $U_{1}, U_{2}$ of $U$ such that any fixed point of $k$ in $U$ is contained in $U_{1} \cup U_{2}$, then $\operatorname{ind}_{X}(k, U)=\operatorname{ind}_{X}\left(k, U_{1}\right)+\operatorname{ind}_{X}\left(k, U_{2}\right)$.

iv) (Excision) Given an open subset $U_{1}$ of $U$ such that $k$ has no fixed points in $U \backslash U_{1}$, then $\operatorname{ind}_{X}(k, U)=$ $\operatorname{ind}_{X}\left(k, U_{1}\right)$.

v) (Commutativity) Let $X$ and $Y$ be metric ANRs. Suppose that $U$ and $V$ are open subsets of $X$ and $Y$ respectively and that $k: U \rightarrow Y$ and $h: V \rightarrow X$ are locally compact maps. Assume that one of the sets of fixed points of $h k$ in $k^{-1}(V)$ or $k h$ in $h^{-1}(U)$ is compact. Then the other set is compact as well and $\operatorname{ind}_{X}\left(h k, k^{-1}(V)\right)=\operatorname{ind}_{Y}\left(k h, h^{-1}(U)\right)$.

vi) (Generalized homotopy invariance) Let $I$ be a compact real interval and $\Omega$ an open subset of $X \times I$. For any $\lambda \in I$, denote $\Omega_{\lambda}=\{x \in X:(x, \lambda) \in \Omega\}$. Let $H: \Omega \rightarrow X$ be a locally compact map such that the set $\{(x, \lambda) \in \Omega: H(x, \lambda)=x\}$ is compact. Then $\operatorname{ind}_{X}\left(H(\cdot, \lambda), \Omega_{\lambda}\right)$ is independent of $\lambda$.

\section{BifuRCATION RESUlTS FOR FIRST ORDER EQUATIONS}

Let $M$ be a compact $\partial$-manifold in $\mathbb{R}^{k}$. Consider the following RFDE

$$
x^{\prime}(t)=f\left(\lambda, t, x_{t}\right),
$$

where $f:[0,+\infty) \times \mathbb{R} \times C((-\infty, 0], M) \rightarrow \mathbb{R}^{k}$ is an inward functional field on $M$ depending on a parameter $\lambda \in[0,+\infty)$, which is $T$-periodic in the second variable ( $T$ being a positive real number).

Suppose that $f$ is bounded on any set $[0, \bar{\lambda}] \times \mathbb{R} \times C((-\infty, 0], M), \bar{\lambda}>0$, and that

$$
f(0, t, \varphi)=0 \quad \text { for any }(t, \varphi) \in \mathbb{R} \times C((-\infty, 0], M) .
$$

Moreover, assume that $f$ verifies the following assumption (compare with assumption (H) on page 4):

$(\tilde{\mathrm{H}})$ Given $\bar{t}>0$ and $\bar{\lambda}>0$, and given any compact brush $Q$ of $C((-\infty, 0], M)$, there exists $L \geq 0$ such that

$$
\|f(\lambda, t, \varphi)-f(\lambda, t, \psi)\| \leq L \sup _{s \leq 0}\|\varphi(s)-\psi(s)\|
$$

for all $t \in[0, \bar{t}], \lambda \in[0, \bar{\lambda}]$, and $\varphi, \psi \in Q$.

We will now prove a global continuation result (Corollary 3.6 below) for the equation (3.1) in the case when the Euler-Poincaré characteristic of $M$ is nonzero. The results of this section, in particular Theorem 3.5 will play a crucial role in the proof of Theorem 4.1 below. 
In the sequel we will adopt the following notation. By $C_{0}([-T, 0], M)$ we shall mean the (complete) metric space of the continuous functions $\psi:[-T, 0] \rightarrow M$ such that $\psi(-T)=\psi(0)$, endowed with the metric induced by the Banach space $C\left([-T, 0], \mathbb{R}^{k}\right)$. Since $M$ is an ANR, it is not difficult to show (see e.g. 9]) that the metric space $C_{0}([-T, 0], M)$ is an ANR as well. We shall denote by $C_{T}(M)$ the set of the continuous $T$-periodic maps from $\mathbb{R}$ to $M$ with the metric induced by the Banach space $C_{T}\left(\mathbb{R}^{k}\right)$ of the continuous $T$-periodic $\mathbb{R}^{k}$-valued maps (with the standard supremum norm).

We will say that $(\lambda, x) \in[0,+\infty) \times C_{T}(M)$ is a $T$-periodic pair of (3.1) if $x: \mathbb{R} \rightarrow M$ is a $T$-periodic solution of (3.1) corresponding to $\lambda$. A $T$-periodic pair of the type $(0, x)$ is said to be trivial. In this case, because of assumption (3.2), the function $x$ is constant.

A pair $(\lambda, \psi) \in[0,+\infty) \times C\left([-T, 0], \mathbb{R}^{k}\right)$ will be called a source pair (of (3.1) ) if there exists $x \in C_{T}(M)$ such that $x(t)=\psi(t)$ for all $t \in[-T, 0]$ and $(\lambda, x)$ is a $T$-periodic pair. Notice that, if $(\lambda, \psi)$ is a source pair, then the map $\psi$ takes values on $M$. A source pair of the type $(0, \psi)$ will be called trivial. In this case the map $\psi$ is a constant $M$-valued map, since it is the restriction of a constant map defined on $\mathbb{R}$.

Observe that the map $\Theta:(\lambda, x) \mapsto(\lambda, \psi)$ which associates to a $T$-periodic pair $(\lambda, x)$ the corresponding source pair $(\lambda, \psi)$ is continuous, $\psi$ being the restriction of $x$ to the interval $[-T, 0]$. Actually, $\Theta$ is an isometry between the set $\Sigma \subseteq[0,+\infty) \times C_{T}(M)$ of the $T$-periodic pairs and the set $S \subseteq[0,+\infty) \times$ $C\left([-T, 0], \mathbb{R}^{k}\right)$ of the source pairs.

It is not difficult to see that $\Sigma$ is closed in $[0,+\infty) \times C_{T}(M)$ (and locally closed in $[0,+\infty) \times C_{T}\left(\mathbb{R}^{k}\right)$ ). It is consequently locally complete, as a closed subset of a locally complete space. Moreover, using Ascoli's Theorem, one can show that $\Sigma$ is actually a locally compact space, and this fact will turn out to be useful in order to get our results.

Given $\varphi \in C_{0}([-T, 0], M)$, we will denote by $\widehat{\varphi}$ the unique element of $C((-\infty, 0], M)$ obtained by considering the $T$-periodic backward extension of the function $\varphi$; i.e. $\widehat{\varphi}$ is defined as follows:

$$
\widehat{\varphi}(\theta)=\varphi(\theta+n T) \quad \text { if } \theta \in[-(n+1) T,-n T], n \in \mathbb{N} .
$$

Observe that $C_{0}([-T, 0], M)$ is bounded and closed as a subset of the Banach space $C\left([-T, 0], \mathbb{R}^{k}\right)$. Hence, $C_{0}([-T, 0], M)$ being an ANR, there exist a bounded open subset $U$ of $C\left([-T, 0], \mathbb{R}^{k}\right)$ containing $C_{0}([-T, 0], M)$ and a retraction $\rho$ of $U$ onto $C_{0}([-T, 0], M)$.

Now, given $\lambda \in[0,+\infty)$, consider the operator

$$
P_{\lambda}: U \rightarrow C\left([-T, 0], \mathbb{R}^{k}\right)
$$

defined as $P_{\lambda}(\psi)(s)=x(s+T)$, where $x$ is the unique solution, ensured by Proposition 2.4. of the following initial value problem:

$$
\begin{cases}x^{\prime}(t)=f\left(\lambda, t, x_{t}\right), & t>0 \\ x(t)=\widehat{\rho(\psi)}(t), & t \leq 0\end{cases}
$$

The following two propositions regard some crucial properties of $P_{\lambda}$. The proof of Proposition 3.1 is straightforward and, therefore, is omitted.

Proposition 3.1. The set of fixed points of $P_{\lambda}$ is contained in $C_{0}([-T, 0], M)$. Moreover, the fixed points of $P_{\lambda}$ correspond to the T-periodic solutions of the equation

$$
x^{\prime}(t)=f\left(\lambda, t, x_{t}\right)
$$

in the following sense: $\psi$ is a fixed point of $P_{\lambda}$ if and only if it is the restriction to $[-T, 0]$ of a T-periodic solution. 
Proposition $3.2([5])$. The map $P:[0,+\infty) \times U \rightarrow C\left([-T, 0], \mathbb{R}^{k}\right)$, defined by $(\lambda, \psi) \mapsto P_{\lambda}(\psi)$, is completely continuous.

Proof. To show that $P$ is continuous, let $\left\{\psi_{n}\right\}$ be a sequence in $U$ which converges to $\psi$, and let $\left\{\lambda_{n}\right\}$ be a sequence in $[0,+\infty)$ converging to $\lambda$. Since $\rho$ is continuous, we have $\rho\left(\psi_{n}\right) \rightarrow \rho(\psi)$. Thus, $\widehat{\rho\left(\psi_{n}\right)}(\theta) \rightarrow$ $\widehat{\rho(\psi)}(\theta)$ uniformly for $\theta \in(-\infty, 0]$.

Now, let $x^{n}: \mathbb{R} \rightarrow M$ be the unique solution (ensured by Proposition 2.4) of the initial value problem

$$
\begin{cases}x^{\prime}(t)=f\left(\lambda_{n}, t, x_{t}\right), & t>0, \\ x(t)=\hat{\rho\left(\psi_{n}\right)}(t), & t \leq 0 .\end{cases}
$$

One can show that every subsequence of $\left\{x^{n}(t)\right\}$ has a subsequence which converges uniformly on compact subintervals of $\mathbb{R}$ to the unique solution $x(t)$ of problem (3.3). Therefore, $x^{n}(t) \rightarrow x(t)$ uniformly on compact subintervals of $\mathbb{R}$ and, consequently, $P\left(\lambda_{n}, \psi_{n}\right) \rightarrow P(\lambda, \psi)$. This shows that the map $P$ is continuous.

To prove that $P$ is completely continuous, it is sufficient to show that, given $b>0$, the image $P([0, b] \times$ $U)$ is compact. This fact follows from Ascoli's Theorem.

Assume now $\lambda=0$. By (3.2), given $\psi \in U$, problem (3.3) becomes

$$
\begin{cases}x^{\prime}(t)=0, & t>0 \\ x(t)=\hat{\rho(\psi)}(t), & t \leq 0\end{cases}
$$

Any solution of this problem for $t \geq 0$ is constantly equal to $\widehat{\rho(\psi)}(0)=\rho(\psi)(0)$. It follows that

$$
P_{0}(\psi)(s)=\rho(\psi)(0), \quad s \in[-T, 0] .
$$

Hence, $P_{0}$ sends $U$ into the subset of the constant $M$-valued functions (which can be identified with $M$ ), and its restriction $\left.P_{0}\right|_{M}: M \rightarrow M$ coincides with the identity $I_{M}$ of $M$. By the commutativity and normalization properties of the fixed point index we get

$$
\operatorname{ind}_{E}\left(P_{0}, U\right)=\operatorname{ind}_{M}\left(P_{0}, M\right)=\Lambda\left(I_{M}\right)=\chi(M),
$$

where $E=C\left([-T, 0], \mathbb{R}^{k}\right)$.

The following topological lemma is needed.

Lemma 3.3 (13]). Let $Z$ be a compact subset of a locally compact metric space $Y$. Assume that any compact subset of $Y$ containing $Z$ has nonempty boundary. Then $Y \backslash Z$ contains a connected set whose closure is not compact and intersects $Z$.

Remark 3.4. Let $(Y, Z)$ be a topological pair satisfying the assumptions of Lemma 3.3, It is not hard to see that, given any compact subset $K$ of $Y$ containing $Z$, the pair $(Y, K)$ verifies the assumptions of Lemma 3.3 as well.

In the sequel, given a metric space $Y$, a subset $G$ of $\mathbb{R} \times Y$ and $\lambda \in \mathbb{R}$, we will denote by $G_{\lambda}$ the slice $\{y \in Y:(\lambda, y) \in G\}$.

Theorem 3.5. Let $M$ be a compact $\partial$-manifold with nonzero Euler-Poincaré characteristic, and let $f:[0,+\infty) \times \mathbb{R} \times C((-\infty, 0], M) \rightarrow \mathbb{R}^{k}$ be an inward parametrized functional field on $M$ which is $T$ periodic in the second variable. Suppose that $f$ is bounded on any set $[0, \bar{\lambda}] \times \mathbb{R} \times C((-\infty, 0], M)$ and verifies condition (3.2) and assumption $(\tilde{H})$. Let

$$
\Sigma=\left\{(\lambda, x) \in[0,+\infty) \times C_{T}(M):(\lambda, x) \text { is a T-periodic pair of (3.1) }\right\} .
$$


Then the pair $\left(\Sigma,\{0\} \times \Sigma_{0}\right)$ verifies the assumptions of Lemma 3.3.

Proof. Let $S \subseteq[0,+\infty) \times C\left([-T, 0], \mathbb{R}^{k}\right)$ be the set of the source pairs of equation (3.1). Recall that, by Proposition 3.1

$$
S=\left\{(\lambda, \psi) \in[0,+\infty) \times U: P_{\lambda}(\psi)=\psi\right\} .
$$

Notice that, actually, $S$ is contained in $[0,+\infty) \times C_{0}([-T, 0], M)$ and, as a consequence of Proposition 3.2. it is locally compact. Moreover, the slice $S_{0}$ coincides with $M$ (regarded as the set of constant functions from $[-T, 0]$ to $M)$.

Let us show that the pair $\left(S,\{0\} \times S_{0}\right)$ verifies the assumptions of Lemma 3.3 Assume, by contradiction, that there exists a compact set $\widehat{S} \subseteq S$ containing $\{0\} \times S_{0}$ and with empty boundary in $S$. Thus, $\widehat{S}$ is also an open subset of the metric space $S$. Hence, there exists a bounded open subset $\Omega$ of $[0,+\infty) \times U$ such

that $\widehat{S}=\Omega \cap S$. Since $\widehat{S}$ is compact, the generalized homotopy invariance property of the fixed point index implies that $\operatorname{ind}_{E}\left(P_{\lambda}, \Omega_{\lambda}\right)$ does not depend on $\lambda \in[0,+\infty)$. Moreover, the slice $\widehat{S}_{\lambda}=\Omega_{\lambda} \cap S_{\lambda}$ is empty for some $\lambda$. This implies that $\operatorname{ind}_{E}\left(P_{\lambda}, \Omega_{\lambda}\right)=0$ for any $\lambda \in[0,+\infty)$ and, in particular, $\operatorname{ind}_{E}\left(P_{0}, \Omega_{0}\right)=0$. Now, since $\Omega_{0}$ is an open subset of $U$ containing $M$, by the excision property of the fixed point index and taking into account equality (3.4), we get that

$$
\operatorname{ind}_{E}\left(P_{0}, \Omega_{0}\right)=\operatorname{ind}_{E}\left(P_{0}, U\right)=\chi(M) \neq 0,
$$

which is a contradiction.

Finally, to prove the assertion observe that the map $\Theta: \Sigma \rightarrow S$, which associates to any $T$-periodic pair $(\lambda, x)$ the corresponding source pair $(\lambda, \psi)$, is a homeomorphism. Moreover, the restriction of $\Theta$ to $\{0\} \times \Sigma_{0}=\{0\} \times M \subseteq \Sigma$ as domain and to $\{0\} \times S_{0}=\{0\} \times M \subseteq S$ as codomain is the identity. Hence, the pair $\left(\Sigma, \Sigma_{0}\right)$ verifies the assumptions of Lemma 3.3 as well.

Corollary 3.6. Let the assumptions of Theorem 3.5 be satisfied. Then, the equation (3.1) admits an unbounded connected set of nontrivial T-periodic pairs whose closure meets the set of the trivial T-periodic pairs. In particular, for any fixed $\lambda$ the equation (3.1) has a T-periodic solution.

Proof. Because of Lemma 3.3. there exists a connected subset $A$ of $\Sigma$ whose closure in $\Sigma$ intersects $\{0\} \times M$ and is not compact. Since $\Sigma$ is a closed subset of $[0,+\infty) \times C_{T}(M)$, the closure $\bar{A}$ of $A$ in $\Sigma$ is the same as in $[0,+\infty) \times C_{T}(M)$. Thus, $\bar{A}$ cannot be bounded since, otherwise, it would be compact because of Ascoli's Theorem. Moreover, since $C_{T}(M)$ is bounded, the set $A$ is necessarily unbounded in $\lambda$. This implies, in particular, that the equation (3.1) has a $T$-periodic solution for any $\lambda \geq 0$.

\section{Bifurcation Results For motion EQUations}

Let $X \subseteq \mathbb{R}^{s}$ be a boundaryless manifold. Given $q \in X$, let $\left(T_{q} X\right)^{\perp} \subseteq \mathbb{R}^{s}$ denote the normal space of $X$ at $q$. Since $\mathbb{R}^{s}=T_{q} X \oplus\left(T_{q} X\right)^{\perp}$, any vector $u \in \mathbb{R}^{s}$ can be uniquely decomposed into the sum of the parallel (or tangential) component $u_{\pi} \in T_{q} X$ of $u$ at $q$ and the normal component $u_{\nu} \in\left(T_{q} X\right)^{\perp}$ of $u$ at q. By

$$
T X=\left\{(q, v) \in \mathbb{R}^{s} \times \mathbb{R}^{s}: q \in X, v \in T_{q} X\right\}
$$

we denote the tangent bundle of $X$, which is a smooth manifold containing a natural copy of $X$ via the embedding $q \mapsto(q, 0)$. The natural projection of $T X$ onto $X$ is just the restriction (to $T X$ as domain and to $X$ as codomain) of the projection of $\mathbb{R}^{s} \times \mathbb{R}^{s}$ onto the first factor.

It is known that, associated with $X \subseteq \mathbb{R}^{s}$, there exists a unique smooth map $R: T X \rightarrow \mathbb{R}^{s}$, called the reactive force (or inertial reaction), with the following properties: 
(a) $R(q, v) \in\left(T_{q} X\right)^{\perp}$ for any $(q, v) \in T X$;

(b) $R$ is quadratic in the second variable;

(c) given $(q, v) \in T X, R(q, v)$ is the unique vector such that $(v, R(q, v))$ belongs to $T_{(q, v)}(T X)$;

(d) any $C^{2}$ curve $\gamma:(a, b) \rightarrow X$ verifies the condition $\gamma_{\nu}^{\prime \prime}(t)=R\left(\gamma(t), \gamma^{\prime}(t)\right)$ for any $t \in(a, b)$, i.e. for each $t \in(a, b)$, the normal component $\gamma_{\nu}^{\prime \prime}(t)$ of $\gamma^{\prime \prime}(t)$ at $\gamma(t)$ equals $R\left(\gamma(t), \gamma^{\prime}(t)\right)$.

Let $F: \mathbb{R} \times C((-\infty, 0], X) \rightarrow \mathbb{R}^{s}$ be a functional field which is $T$-periodic in the first variable. Consider the following retarded functional motion equation on $X$, depending on a parameter $\lambda \geq 0$ :

$$
x_{\pi}^{\prime \prime}(t)=\lambda\left(F\left(t, x_{t}\right)-\varepsilon x^{\prime}(t)\right),
$$

where $x_{\pi}^{\prime \prime}(t)$ stands for the parallel component of the acceleration $x^{\prime \prime}(t) \in \mathbb{R}^{s}$ at the point $x(t)$, and $\varepsilon \geq 0$ is the frictional coefficient. By properties (a) and (d) above, equation (4.1) can be equivalently written as

$$
x^{\prime \prime}(t)=R\left(x(t), x^{\prime}(t)\right)+\lambda\left(F\left(t, x_{t}\right)-\varepsilon x^{\prime}(t)\right) .
$$

Given $\lambda \geq 0$, equation (4.2) is equivalent to the RFDE

$$
\left\{\begin{array}{l}
x^{\prime}(t)=y(t) \\
y^{\prime}(t)=R(x(t), y(t))+\lambda\left(F\left(t, x_{t}\right)-\varepsilon y(t)\right)
\end{array}\right.
$$

in the following sense: a function $x: J \rightarrow M$ is a solution of (4.2) if and only if the pair $\left(x, x^{\prime}\right)$ is a solution of (4.3). Let us stress that system (4.3) is actually a RFDE, which can be written as

$$
\left(x^{\prime}(t), y^{\prime}(t)\right)=G\left(\lambda, t,\left(x_{t}, y_{t}\right)\right),
$$

where the map $G:[0,+\infty) \times \mathbb{R} \times C((-\infty, 0], T X) \rightarrow \mathbb{R}^{s} \times \mathbb{R}^{s}$ is defined by

$$
G(\lambda, t,(\varphi, \psi))=(\psi(0), R(\varphi(0), \psi(0))+\lambda(F(t, \varphi)-\varepsilon \psi(0))) .
$$

We remark that $G$ is a parametrized functional field on $T X$. Indeed, the condition

$$
G(\lambda, t,(\varphi, \psi)) \in T_{(\varphi(0), \psi(0))} T X
$$

is verified for all $(\lambda, t,(\varphi, \psi)) \in[0,+\infty) \times \mathbb{R} \times C((-\infty, 0], T X)$ (see, for example, [10 for more details).

Observe that, if equation (4.2) reduces to the so-called inertial equation

$$
x^{\prime \prime}(t)=R\left(x(t), x^{\prime}(t)\right),
$$

one obtains the geodesics of $X$ as solutions.

Given $\lambda \geq 0$, any $T$-periodic solution of (4.1) is called a forced oscillation corresponding to the value $\lambda$ of the parameter. In other words, a forced oscillation is a solution of the differential equation (4.1) which belongs to $C_{T}^{1}(X)$, the metric subspace of the Banach space $C_{T}^{1}\left(\mathbb{R}^{s}\right)$ of the $T$-periodic $C^{1}$ maps $x: \mathbb{R} \rightarrow X$.

We will say that $(\lambda, x) \in[0,+\infty) \times C_{T}^{1}(X)$ is a $T$-forced pair of (4.1) if $x: \mathbb{R} \rightarrow X$ is a forced oscillation of (4.1) corresponding to $\lambda$. That is, $(\lambda, x) \in[0,+\infty) \times C_{T}^{1}(X)$ is a $T$-forced pair of (4.1) if and only if $\left(\lambda,\left(x, x^{\prime}\right)\right) \in[0,+\infty) \times C_{T}(T X)$ is a $T$-periodic pair of system (4.3).

Denote by $\Gamma$ the closed subset of $[0,+\infty) \times C_{T}^{1}(X)$ of all the $T$-forced pairs of (4.1). Notice that, by Ascoli's Theorem, the set $\Gamma$ is locally compact. Given $q \in X$, we denote by $\bar{q} \in C_{T}^{1}(X)$ the constant map $t \mapsto q, t \in \mathbb{R}$. We will call trivial $T$-forced pair a pair of the form $(0, \bar{q}), q \in X$, and we will say that $X$ is the trivial solutions' manifold of $\Gamma$.

An element $q_{0} \in X$ will be called a bifurcation point of the equation (4.1) if every neighborhood of $\left(0, \bar{q}_{0}\right)$ in $[0,+\infty) \times C_{T}^{1}(X)$ contains a nontrivial $T$-forced pair, i.e. an element of $\Gamma \backslash X$. One can show 
that any nontrivial $T$-forced pair $(\lambda, x)$ of (4.1), sufficiently close to $\left(0, \bar{q}_{0}\right)$, must have $\lambda>0$. This is due to the fact that the solutions of the inertial equation are geodesics of $X$, and there are no nontrivial closed geodesics too close to a given point in a Riemannian manifold.

We are now in the position to state our main result.

Theorem 4.1. Let $X \subseteq \mathbb{R}^{s}$ be a compact boundaryless manifold whose Euler-Poincaré characteristic $\chi(X)$ is different from zero, and $F: \mathbb{R} \times C((-\infty, 0], X) \rightarrow \mathbb{R}^{s}$ a functional field which is T-periodic in the first variable. Suppose that $F$ is bounded and verifies (H), and let $\varepsilon \geq 0$ be given. Then, the equation (4.1) admits an unbounded connected set of nontrivial $T$-forced pairs whose closure meets the set of the trivial $T$-forced pairs at some bifurcation point. If, in addition, $\varepsilon$ is positive, then the bifurcating branch is necessarily unbounded with respect to $\lambda$.

Proof. Step 1. Assume first $\varepsilon>0$, and consider the following system:

$$
\left\{\begin{array}{l}
x^{\prime}(t)=\alpha(\mu) y(t), \\
y^{\prime}(t)=\alpha(\mu)(R(x(t), y(t))-\varepsilon(1-\alpha(\mu)) y(t))+\beta(\mu)\left(F\left(t, x_{t}\right)-\varepsilon y(t)\right),
\end{array}\right.
$$

where

$$
\alpha(\mu)= \begin{cases}\mu & \text { if } 0 \leq \mu \leq 1 \\ 1 & \text { if } \mu \geq 1\end{cases}
$$

and

$$
\beta(\mu)= \begin{cases}0 & \text { if } 0 \leq \mu \leq 1, \\ \mu-1 & \text { if } \mu \geq 1\end{cases}
$$

In this way, when $0 \leq \mu \leq 1$ we get

$$
\left\{\begin{array}{l}
x^{\prime}(t)=\mu y(t) \\
y^{\prime}(t)=\mu(R(x(t), y(t))-\varepsilon(1-\mu) y(t))
\end{array}\right.
$$

and when $\mu \geq 1$ we get

$$
\left\{\begin{aligned}
x^{\prime}(t) & =y(t) \\
y^{\prime}(t) & =R(x(t), y(t))+(\mu-1)\left(F\left(t, x_{t}\right)-\varepsilon y(t)\right) .
\end{aligned}\right.
$$

Define $G:[0,+\infty) \times \mathbb{R} \times C((-\infty, 0], T X) \rightarrow \mathbb{R}^{s} \times \mathbb{R}^{s}$ by

$$
G(\mu, t,(\varphi, \psi))=(\alpha(\mu) \psi(0), \alpha(\mu)(R(\varphi(0), \psi(0))-\varepsilon(1-\alpha(\mu)) \psi(0))+\beta(\mu)(F(t, \varphi)-\varepsilon \psi(0))) .
$$

Clearly, $G$ is a parametrized $T$-periodic functional field on $T X$, which verifies assumption $(\tilde{\mathrm{H}})$ since $F$ verifies $(\mathrm{H})$.

Given $c>0$, set

$$
M_{c}=\{(q, v) \in T X:\|v\| \leq c\} .
$$

It is not difficult to show that $M_{c} \subseteq T X$ is a compact $\partial$-manifold in $\mathbb{R}^{s} \times \mathbb{R}^{s}$ with boundary

$$
\partial M_{c}=\left\{(q, v) \in M_{c}:\|v\|=c\right\} .
$$

Let $G_{c}$ be the restriction of the map $G$ to $[0,+\infty) \times \mathbb{R} \times C\left((-\infty, 0], M_{c}\right)$. Clearly, $G_{c}$ is a parametrized $T$-periodic functional field on $M_{c}$ which verifies $(\tilde{\mathrm{H}})$ since so does $G$. Moreover, given any $\mu_{0}>0$, the map $G_{c}$ is bounded on $\left[0, \mu_{0}\right] \times \mathbb{R} \times C\left((-\infty, 0], M_{c}\right)$. To see this notice that $0 \leq \alpha(\mu) \leq 1$ and $0 \leq \beta(\mu) \leq \beta\left(\mu_{0}\right)$. Now, the map $F$ is bounded by assumption, hence there is $K$ such that $\|F(t, \varphi)\| \leq K$ 
for all $(t, \varphi)$. Moreover, the compactness of $M_{c}$ implies that there is $K_{1}$ such that $\|R(q, v)\| \leq K_{1}$ for any $(q, v) \in M_{c}$. Therefore, given $(\mu, t,(x, y)) \in\left[0, \mu_{0}\right] \times \mathbb{R} \times C\left((-\infty, 0], M_{c}\right)$, one has $\|\alpha(\mu) \psi(0)\| \leq c$,

$$
\|\alpha(\mu)(R(\varphi(0), \psi(0))-\varepsilon(1-\alpha(\mu)) \psi(0))\| \leq K_{1}+\varepsilon c
$$

and

$$
\|\beta(\mu)(F(t, \varphi)-\varepsilon \psi(0))\| \leq \beta\left(\mu_{0}\right)(K+\varepsilon c) .
$$

This shows that $G_{c}$ is bounded on $\left[0, \mu_{0}\right] \times \mathbb{R} \times C\left((-\infty, 0], M_{c}\right)$, being the sum of bounded maps.

We claim that, if $c>0$ is large enough, then $G_{c}$ is inward on $M_{c}$. To see this, observe that the tangent cone of $M_{c}$ at $(q, v) \in \partial M_{c}$ is the half subspace of $T_{(q, v)} M_{c}$ given by

$$
C_{(q, v)} M_{c}=\left\{(\dot{q}, \dot{v}) \in T_{(q, v)}(T X):\langle v, \dot{v}\rangle \leq 0\right\},
$$

where $\langle\cdot, \cdot\rangle$ denotes the inner product in $\mathbb{R}^{s}$. Thus we have to show that, if $c>0$ is large enough, then $G_{c}(\mu, t,(\varphi, \psi))$ belongs to $C_{(\varphi(0), \psi(0))} M_{c}$ for any $\mu \in[0,+\infty), t \in \mathbb{R}$, and any pair $(\varphi, \psi) \in C\left((-\infty, 0], M_{c}\right)$ such that $(\varphi(0), \psi(0)) \in \partial M_{c}$. In other words, we need to prove that for any $\mu, t$ and any pair $(\varphi, \psi)$ such that $\|\psi(0)\|=c$, with $c$ to be chosen, we have

$$
\langle\psi(0), \alpha(\mu)(R(\varphi(0), \psi(0))-\varepsilon(1-\alpha(\mu)) \psi(0))+\beta(\mu)(F(t, \varphi)-\varepsilon \psi(0))\rangle \leq 0 .
$$

That is,

$$
\alpha(\mu)(\langle\psi(0), R(\varphi(0), \psi(0))\rangle-\varepsilon(1-\alpha(\mu))\langle\psi(0), \psi(0)\rangle)+\beta(\mu)(\langle\psi(0), F(t, \varphi)\rangle-\varepsilon\langle\psi(0), \psi(0)\rangle) \leq 0 .
$$

Observe first that $\langle\psi(0), R(\varphi(0), \psi(0))\rangle=0$ since $R(\varphi(0), \psi(0))$ belongs to $\left(T_{\varphi(0)} X\right)^{\perp}$ and that, clearly, $\langle\psi(0), \psi(0)\rangle=c^{2}$. Assume now $0 \leq \mu \leq 1$. Then, $\alpha(\mu)=\mu$ and $\beta(\mu)=0$, and thus

$$
\langle\psi(0), \alpha(\mu)(R(\varphi(0), \psi(0))-\varepsilon(1-\alpha(\mu)) \psi(0))\rangle=-\varepsilon \mu(1-\mu) c^{2} \leq 0 .
$$

On the other hand, when $\mu \geq 1$ one has $\alpha(\mu)=1$ and $\beta(\mu)=\mu-1$. In addition, one has

$$
\langle\psi(0), F(t, \varphi)\rangle \leq\|\psi(0)\|\|F(t, \varphi)\| \leq K\|\psi(0)\|,
$$

recalling that $K$ is such that $\|F(t, \varphi)\| \leq K$ for all $(t, \varphi) \in \mathbb{R} \times C((-\infty, 0], X)$. Consequently,

$\langle\psi(0), \alpha(\mu) R(\varphi(0), \psi(0))+\beta(\mu)(F(t, \varphi)-\varepsilon \psi(0))\rangle=(\mu-1)\langle\psi(0), F(t, \varphi)-\varepsilon \psi(0)\rangle \leq(\mu-1)\left(K c-\varepsilon c^{2}\right)$.

This shows that, if we choose $c \geq K / \varepsilon$, then $G_{c}$ is an inward parametrized functional field on $M_{c}$, as claimed (here the condition $\varepsilon>0$ is crucial).

Let now

$$
\Sigma=\left\{(\mu,(x, y)) \in[0,+\infty) \times C_{T}\left(M_{c}\right):(\mu,(x, y)) \text { is a } T \text {-periodic pair of (4.4) }\right\} .
$$

Observe that the slice $\Sigma_{0}$ coincides with $M_{c}$. Let us show that $\Sigma_{\mu}=X \times\{0\}$ for $0<\mu<1$. To see this recall that, when $0<\mu<1$, system (4.4) becomes

$$
\left\{\begin{array}{l}
x^{\prime}(t)=\mu y(t) \\
y^{\prime}(t)=\mu(R(x(t), y(t))-\varepsilon(1-\mu) y(t)) .
\end{array}\right.
$$

Let $\delta(t)=\|y(t)\|^{2}, t \in \mathbb{R}$. One has

$$
\delta^{\prime}(t)=2\left\langle y(t), y^{\prime}(t)\right\rangle=2 \mu\langle y(t),(R(x(t), y(t))-\varepsilon(1-\mu) y(t))\rangle=-2 \varepsilon \mu(1-\mu)\|y(t)\|^{2}=-a \delta(t),
$$

where $a=2 \varepsilon \mu(1-\mu)>0$. This proves that if $(\mu,(x, y))$ is a $T$-periodic pair with $0<\mu<1$, then $y(t)=0$ for any $t$. That is, $(x, y)$ belongs to $X \times\{0\}$. 
Consider the compact set

$$
\Upsilon=\left(\{0\} \times M_{c}\right) \cup([0,1] \times X \times\{0\}) \subseteq \Sigma .
$$

We claim that the pair $(\Sigma, \Upsilon)$ verifies the assumptions of Lemma 3.3. To this end, observe first that $\chi\left(M_{c}\right)=\chi(X)$ since $M_{c}$ and $X$ are homotopically equivalent ( $X$ being a deformation retract of $\left.T X\right)$. Consequently, since $\chi(X) \neq 0$ by assumption, we get $\chi\left(M_{c}\right) \neq 0$. Thus, given $c>K / \varepsilon$, we can apply Theorem 3.5 with $M=M_{c}$ and $f=G_{c}$, and we get that the pair $\left(\Sigma,\{0\} \times \Sigma_{0}\right)$ verifies the assumptions of Lemma 3.3. Hence, being $\Upsilon$ a compact subset of $\Sigma$ containing $\{0\} \times \Sigma_{0}=\{0\} \times M_{c}$, by Remark 3.4 the pair $(\Sigma, \Upsilon)$ verifies the assumptions of Lemma 3.3 as claimed. Hence, there exists a connected subset $A$ in $\Sigma \backslash \Upsilon$ whose closure in $\Sigma$ intersects $\Upsilon$ and is not compact.

Recall that, for $\mu \geq 1$, system (4.4) becomes

$$
\left\{\begin{array}{l}
x^{\prime}(t)=y(t) \\
y^{\prime}(t)=R(x(t), y(t))+(\mu-1)\left(F\left(t, x_{t}\right)-\varepsilon y(t)\right) .
\end{array}\right.
$$

Clearly, up to the change of variable $\lambda=\mu-1$, this coincides with system (4.3), which is equivalent to equation (4.1). The above argument shows that system (4.3) admits an unbounded connected set $A \subseteq[0,+\infty) \times C_{T}\left(M_{c}\right)$ of nontrivial $T$-periodic pairs whose closure in $[0,+\infty) \times C_{T}\left(M_{c}\right)$, which is the same as in $[0,+\infty) \times C_{T}(T X)$, intersects $\{0\} \times C_{T}(T X)$ in a nonempty subset of $\{0\} \times X \times\{0\}$. Notice that $A$ is necessarily unbounded with respect to $\lambda$, being $C_{T}\left(M_{c}\right)$ a bounded metric space.

Finally, to prove the assertion in the case $\varepsilon>0$, consider the map $\Pi:[0,+\infty) \times C_{T}(T X) \rightarrow[0,+\infty) \times$ $C_{T}^{1}(X)$ associating to a $T$-periodic pair $(\lambda,(x, y))$ of system (4.3) the $T$-forced pair $(\lambda, x)$ of equation (4.1). Observe that $\Pi$ is continuous with inverse given by $(\lambda, x) \mapsto\left(\lambda,\left(x, x^{\prime}\right)\right)$. Moreover, the restriction of $\Pi$ to $\{0\} \times X \times\{0\}$ as domain and to $\{0\} \times X$ as codomain can be regarded as the identity of $X$. Hence $B=\Pi(A)$ is an unbounded connected set of nontrivial $T$-forced pairs whose closure in $[0,+\infty) \times C_{T}^{1}(X)$ meets the set of the trivial $T$-forced pairs. Moreover, $B$ is clearly unbounded with respect to $\lambda$ since so is $A$.

Step 2. Assume now $\varepsilon=0$. Apply Lemma 3.3 to the pair $(\Gamma,\{0\} \times X)$, where $\Gamma$ denotes the set of the $T$-forced pairs of equation

$$
x_{\pi}^{\prime \prime}(t)=\lambda F\left(t, x_{t}\right) .
$$

As already pointed out, $\Gamma$ is a closed, locally compact subset of $[0,+\infty) \times C_{T}^{1}(X)$. Assume, by contradiction, that there exists a compact set $\widehat{\Gamma} \subseteq \Gamma$ containing $\{0\} \times X$ and with empty boundary in the metric space $\Gamma$. Thus, $\widehat{\Gamma}$ is also an open subset of $\Gamma$ and, consequently, $\Gamma \backslash \widehat{\Gamma}$ is closed in $[0,+\infty) \times C_{T}^{1}(X)$. Hence, there exists a bounded open subset $W$ of $[0,+\infty) \times C_{T}^{1}(X)$ such that $\widehat{\Gamma} \subseteq W$ and $\partial W \cap \Gamma=\emptyset$.

Let now $\left\{\varepsilon_{n}\right\}$ be a sequence such that $\varepsilon_{n}>0$ and $\varepsilon_{n} \rightarrow 0$. Given any $n \in \mathbb{N}$, let $\Gamma_{n}$ denote the set of the $T$-forced pairs of the equation

$$
x_{\pi}^{\prime \prime}(t)=\lambda\left(F\left(t, x_{t}\right)-\varepsilon_{n} x^{\prime}(t)\right) .
$$

Since $W$ is bounded and contains $\{0\} \times X$, as a consequence of Step 1 , for any $n \in \mathbb{N}$ there exists a pair $\left(\lambda_{n}, x^{n}\right) \in \Gamma_{n} \cap \partial W$. We may assume $\lambda_{n} \rightarrow \lambda_{0}$ and, by Ascoli's Theorem, $x^{n} \rightarrow x^{0}$ in $C_{T}^{1}(X)$. Thus, $x^{0}$ is a $T$-periodic solution of the equation

$$
x_{\pi}^{\prime \prime}(t)=\lambda_{0} F\left(t, x_{t}\right) .
$$

That is, $\left(\lambda_{0}, x^{0}\right)$ is a $T$-forced pair of (4.5) and, consequently, $\left(\lambda_{0}, x^{0}\right)$ belongs to $\partial W \cap \Gamma$, which is a contradiction. Therefore, by Lemma 3.3 one can find a connected branch $B$ of nontrivial $T$-forced pairs of (4.5) whose closure in $\Gamma$ (which is the same as in $[0,+\infty) \times C_{T}^{1}(X)$ ) intersects $\{0\} \times X$ and is not 
compact. Finally, B cannot be bounded since, otherwise, because of Ascoli's Theorem, its closure would be compact. This completes the proof.

As a straightforward consequence of Theorem 4.1 we get the following existence result (see [5]).

Corollary 4.2. Let $X$ and $F$ be as in Theorem 4.1 and let $\varepsilon>0$. Then, the equation

$$
x_{\pi}^{\prime \prime}(t)=F\left(t, x_{t}\right)-\varepsilon x^{\prime}(t)
$$

has a T-periodic solution.

As we already pointed out in the Introduction, when $\varepsilon=0$, we don't know whether or not the equation

$$
x_{\pi}^{\prime \prime}(t)=F\left(t, x_{t}\right)
$$

has a forced oscillation. Actually, we proved that (4.1) admits an unbounded bifurcating branch, but we cannot assert that such a branch is unbounded with respect to $\lambda$. As far as we know, the problem of the existence of forced oscillations of (4.7) is still open, even in the undelayed situation. In the particular case of the spherical pendulum, i.e. $X=S^{2}$, the existence of forced oscillations for equations without delay has been proved by the last two authors in [12], and this result has been extended in [14] to the case $X=S^{2 n}$.

\section{REFERENCES}

[1] P. Benevieri, A. Calamai, M. Furi, and M.P. Pera, Global branches of periodic solutions for forced delay differential equations on compact manifolds, J. Differential Equations 233 (2007), 404-416.

[2] P. Benevieri, A. Calamai, M. Furi, and M.P. Pera, Forced oscillations for delay motion equations on manifolds, Electron. J. Diff. Eqns. 2007 (2007), No. 62, 1-5.

[3] P. Benevieri, A. Calamai, M. Furi, and M.P. Pera, On forced fast oscillations for delay differential equations on compact manifolds, J. Differential Equations 246 (2009), 1354-1362.

[4] P. Benevieri, A. Calamai, M. Furi, and M.P. Pera, Delay differential equations on manifolds and applications to motion problems for forced constrained systems, to appear on Zeitschrift für Analysis und ihre Anwendungen.

[5] P. Benevieri, A. Calamai, M. Furi, and M.P. Pera, Retarded functional differential equations on manifolds and applications to motion problems for forced constrained systems, Adv. Nonlinear Stud. 9 (2009), 199-214.

[6] R.F. Brown, The Lefschetz fixed point theorem, Scott, Foresman and Co., Glenview, Ill.-London, 1971.

[7] G. Bouligand, Introduction à la géométrie infinitésimale directe, Gauthier-Villard, Paris, 1932.

[8] A. Dold, Lectures on algebraic topology, Springer-Verlag, Berlin, 1972.

[9] J. Eells and G. Fournier, La théorie des points fixes des applications à itérée condensante, Bull. Soc. Math. France 46 (1976), 91-120.

[10] M. Furi, Second order differential equations on manifolds and forced oscillations, Topological Methods in Differential Equations and Inclusions, A. Granas and M. Frigon Eds., Kluwer Acad. Publ. series C, vol. 472, 1995.

[11] M. Furi and M.P. Pera, On the existence of forced oscillations for the spherical pendulum, Boll. Un. Mat. Ital. (7) 4-B (1990), 381-390.

[12] M. Furi and M.P. Pera, The forced spherical pendulum does have forced oscillations. Delay differential equations and dynamical systems (Claremont, CA, 1990), 176-182, Lecture Notes in Math., 1475, Springer, Berlin, 1991.

[13] M. Furi and M.P. Pera, A continuation principle for periodic solutions of forced motion equations on manifolds and applications to bifurcation theory, Pacific J. Math. 160 (1993), 219-244.

[14] M. Furi and M.P. Pera, On the notion of winding number for closed curves and applications to forced oscillations on even-dimensional spheres, Boll. Un. Mat. Ital. (7), 7-A (1993), 397-407.

[15] R. Gaines and J. Mawhin, Coincidence degree and nonlinear differential equations, Lecture Notes in Math., 568, Springer, Berlin, 1977.

[16] A. Granas, The Leray-Schauder index and the fixed point theory for arbitrary ANR's, Bull. Soc. Math. France 100 (1972), 209-228.

[17] V. Guillemin and A. Pollack, Differential Topology, Prentice-Hall Inc., Englewood Cliffs, New Jersey, 1974. 
[18] J.K. Hale and S.M. Verduyn Lunel, Introduction to Functional Differential Equations, Springer Verlag, New York, 1993.

[19] M.W. Hirsch, Differential Topology, Graduate Texts in Math., Vol. 33, Springer Verlag, Berlin, 1976.

[20] J. Leray and J. Schauder, Topologie et équations fonctionnelles, Ann. Sci. École Norm. Sup. 51 (1934), 45-78.

[21] J. Mallet-Paret, R.D. Nussbaum and P. Paraskevopoulos, Periodic solutions for functional-differential equations with multiple state-dependent time lags, Topol. Methods Nonlinear Anal. 3 (1994), 101-162.

[22] J.M. Milnor, Topology from the differentiable viewpoint, Univ. Press of Virginia, Charlottesville, 1965.

[23] J.R. Munkres, Elementary Differential Topology, Princeton University Press, Princeton, New Jersey, 1966.

[24] R.D. Nussbaum, The fixed point index for local condensing maps, Ann. Mat. Pura Appl. 89 (1971), $217-258$.

[25] R.D. Nussbaum, Periodic solutions of some nonlinear autonomous functional differential equations, Ann. Mat. Pura Appl. 101 (1974), 263-306.

[26] R.D. Nussbaum, The fixed point index and fixed point theorems, Topological methods for ordinary differential equations (Montecatini Terme, 1991), 143-205, Lecture Notes in Math., 1537, Springer, Berlin, 1993.

[27] W.M. Oliva, Functional differential equations on compact manifolds and an approximation theorem, J. Differential Equations 5 (1969), 483-496.

[28] W.M. Oliva, Functional differential equations-generic theory. Dynamical systems (Proc. Internat. Sympos., Brown Univ., Providence, R.I., 1974), Vol. I, pp. 195-209. Academic Press, New York, 1976.

[29] E. Spanier, Algebraic Topology, Mc Graw-Hill Series in High Math., New York, 1966.

Pierluigi Benevieri, Massimo Furi, and Maria Patrizia Pera,

Dipartimento di Matematica Applicata "Giovanni Sansone",

Università degli Studi di Firenze

Via S. Marta 3

I-50139 Firenze, Italy

Alessandro Calamai

Dipartimento di Scienze Matematiche

Università Politecnica delle Marche

Via Brecce Bianche

I-60131 Ancona, Italy.

e-mail addresses:

pierluigi.benevieri@unifi.it

calamai@dipmat. univpm. it

massimo.furi@unifi.it

mpatrizia.pera@unifi.it 